\title{
Argentinean's Plants with Interest in Ethnomedicine as Wormers
}

\author{
Haag Griselda ${ }^{1, a}$, Marin Gustavo Horacio ${ }^{2, b^{*}}$ and Errecalde Jorge ${ }^{1, c}$ \\ ${ }^{1}$ Faculty of Medicine, National University of La Plata, \\ Calle 60 y 120 (1900) La Plata, Argentina \\ ${ }^{2}$ CONICET 60 y 120 (1900) La Plata, Argentina \\ a griseldahaag@gmail.com, b gmarin2009@gmail.com, ${ }^{c}$ jerrecalde@yahoo.com.ar
}

Keywords: Ethnomedicine, Argentina, Wormers.

\begin{abstract}
Plants are different all over the world and its indications change according to the country they are located. This paper is developed in order to perform a comprehensive literature's review about plants used as anti-parasitic either in traditional or popular medicine in Argentina, and to provide a scientific support for their use in this medical indication. All Ethnobotany, Ethnomedicine, Phytomedicine books from Libraries of the Faculty of Exact Sciences Faculty of Medical Sciences, Agricultural Science, from all National Universities in Argentina were included in the study. Herbal characteristics and therapies were searched in online databases such as Medline PubMed, Science Direct and Scopus. The results obtained show the potential of Ethnomedicine in Argentina as a source of active ingredients for phytotherapy, providing data about species for antiparasitic treatment. Thus, we found many Argentinean's plants with anti-parasitic properties which might be considered alternatives for natural treatment or future drug development against wormers.
\end{abstract}

\section{Introduction}

Traditionally plants coming from each geographic region of the world had been used for medical purposes for several centuries. This knowledge has been maintained through oral transmission from generation to generation [1].

But this information is rapidly disappearing due to the adoption of measures from new modern culture that displaced the traditional wisdom.

The importance of documenting this knowledge derived from technological changes, or people's socio-economic features, which led to a disuse or total loss of traditional knowledge [2]. Noteworthy, this interest in herbal medicine has increased in the last years.

Pharmaceutical companies focus their attention in the research of new molecules, since classical treatments usually develop resistance. This is the case of anti-parasitic drugs. The last decades were marked by a progressive increase in drug resistance and in rising of the costs of therapies. The research and development of new knowledge about herbs is not longer an option but a necessity.

Herbal medicine then poses as one of the best options for the control of disease resistant to conventional drugs [3].

Medicinal plants are defined by the World Health Organization (WHO) as "any plant species in which all or a portion of it has pharmacological activity" or by the Pharmacopoeia VII edition 2002 first volume (FA 7) - plants or ground whole or ground parts (flowers, fruits, seeds, tubers, bark, etc.) fresh or dried and juices, gums, latex, essential oils and other fixed or similar components, which are used pure or mixed on drug development " [4,5].

The use of coded free vegetable or dispensing medicinal purposes is an established practice in Argentina and has been significantly increased in the past 3 decades [6]. Medicinal plants in Argentina used for therapeutic purposes are included in drug's category; therefore it becomes necessary to guarantee their quality, safety, and effectiveness [6]. Consequently, both health authorities and users needs information concerning about safety and quality of herbal medicines [4] and, in order to ensure continuous, affordable and sustainable supply of medicinal plant materials, it is suitable to demand good quality publication on medicinal plants [5]. 
In Argentina, parasites are considered a severe problem. It exist frequent resistance for classic antiparasitic treatment. On the other hand, Ethnomedicine studies are still scarce. Therefore it is demanded to count with Ethnomedicine information as a starting point for future studies and for Pharmacognosy and Pharmacologic trials, in order to justify their use. In this sense, the World Health Organization itself recognizes the important dimension of medicinal plants as a therapeutic resource, especially in Primary Health Care, existing thereon documents issued by this entity such as monographs, standards of practice of growing and processing products as well as the basis for clinical trials with herbal medicines [5].

Perhaps the inability to patent these products does not motivate the pharmaceutical industry to focus its immense economic flow to them. However, it is also true that the pharmaceutical industry itself is today a true crossroads, unable to find or synthesize all new products the Society needs, so, rediscover new uses for these old natural molecules is not so bad idea. This leds into take a new look about the industry to this type of resource, where there are thousands of undiscovered properties of not so well known regional plants. In the work, we explored the available data of herbs with parasiticide properties, located in Argentina.

\section{Material and Methods}

\section{Geographical area of the study.}

The study area encompasses Argentina territory. The country develops in a physical environment with great diversity of customs, impacting directly on the diversity of plants and treatments; along with a wealth of behavior you inherit immigrants.

It was also considered for this paper that Argentina is a country with a variety of climates, regions, vegetation and diversity of practice in the medical use.

\section{Collection of information}

The work has been carried out by the search and bibliographic review of the literature on Ethnomedicine, ethnoveterinary, Ethnobotany, Pharmacognosy and Phytomedicine topics. Only it has been collected herbal data that had explicit references to it use as antiparasitic, insecticides and insect repellents, discarding all those uses related to magical or superstitious nature or those refered to its use as antiparasitic purgative laxative effect such as gel Aloe vera, Rhamnus frangula bark L., R. Prussian D. L., or oil from Ricinus communis.

\section{Data Sources}

Data about the antiparasitic effects of the herbs studied was obtained exploring Scopus data base, Pubmed, Phytomedicines and Pharmacognosy and Phytotherapy books (either from libraries or on line versions). Formulary Prescription gathering medicinal herbs with antiparasitic uses was extracted from scientific community database.

\section{Results}

\section{Species and botanical families}

The data concerning species and families is shown in Table 1. However complete information including plant's features and indications is shown in table 2. This data reflect the use of species belonging to 25 families, knowing that many species belongs to few families (Figure 1).

50 species belonging to 25 families were studied. Only 4 of them were found in the official Pharmacopeia Drug Edition in Argentina. 12 of the plants studied are banned because of their toxicity and 4 are considered highly toxic but its use is still allowed. Considering the popular knowledge and alternative practices gave us a better understanding of human interactions with their local environment and helps us to obtained appropriate data for validation of traditional uses. 
Table 1. Families Names

\begin{tabular}{|c|c|c|}
\hline Scientific name & Local common name & Family \\
\hline Allium sativum $L$. & Garlic & Liliáceae \\
\hline Artemisina abrotamum L. & Abrótano male & Asteraceae \\
\hline Artemisia absintium & Wormwood & Asteraceae \\
\hline Artemisia matitima $\mathrm{L}$ & Wormwood Maritime & Asteraceae \\
\hline Artemisia mendozana D.C & Wormwood Mendozino & Asteraceae \\
\hline Artemisa vulgaris, $A$. herba & Artemis & Asteraceae \\
\hline Asclepia curassavica & Spanish flag & Asclepiadácea \\
\hline Atractylis gummifera $\mathrm{L}$ & League & Asteraceae \\
\hline Castela erecta ssp & Chaparro bitter & Lorantáceae \\
\hline Chenopodium ambrosioides L. & Quenopodio, Piako, & Amaranthacea \\
\hline Cinchona sp. & Quina & Rubiaceae \\
\hline Combretum micronthum $\mathrm{L}$ & Combreto & Combretaceae \\
\hline Cominum cyminum L. & Cumin & Umbelíferas \\
\hline Curcubita pepo L. & Pumpkin & Cucurbitáceae \\
\hline Daucus carota & Carrot & Umbelíferas \\
\hline Delphinium staphisagria L. & Kills lice, swallow foot & Ranunculaceae \\
\hline Eucalyptus globulus & Eucalyptus & Myrtaceae \\
\hline Eugenia caryophyllus & Clove & Myrtaceae \\
\hline Eugenia uniflora & Pitanga & Myrtaceae \\
\hline Eupatorium cannabium L & Cañamo & Asteraceae \\
\hline Hordeum vulgare L. & Barley & Poaceae \\
\hline Hydrastis canadensis & Goldenseal & Ranunculaceae \\
\hline Inula helenium $\mathrm{L}$. & Elecampane, Grass Moor & Asteraceae \\
\hline Juglans regia & Walnut & Asteraceae \\
\hline Matricaria chamomilla & Chamomile & Asteraceae \\
\hline Melaileuca cajeputi Powell & Cayeput & Myrtaceae \\
\hline Melaleuca leucadendron & Niaouli,Cajeput, & Myrtaceae \\
\hline Mentha Piperita & Mint & Lamiaceae \\
\hline Mentha pulegium L. & European pennyroyal & Lamiaceae \\
\hline Mentha spicata & Peppermint & Lamiaceae \\
\hline Myroxylon balsamiferum & Balsam of Peru & Fabaceae \\
\hline Nephrodium & Bracken fern & Polipodiáceas \\
\hline Nicotiana glauca Graham & Palán-Palán & Solanaceae \\
\hline Nicotiana tabacum & Tobacco & Solanaceae \\
\hline Petiveria alliacea & Bulll grass & Phytolaccaceae \\
\hline Pimpinella anisum L. & Green anise & Umbelíferas \\
\hline Podophyllum peltatum L. & Podophyllum & Berberidáceae \\
\hline Portulaca oleracea L. & Purslane & Portulacaceae \\
\hline Punica granatum $\mathrm{L}$ & Pomegranate & Lythraceae \\
\hline Quassia amara & Quassia bitter, bitter post & Simaroubaceae \\
\hline Rosa gallica L. & Rosebush & Rosáceas \\
\hline Ruta chalepensis & Ruda & Rutaceae \\
\hline Santolina chamaecyparissus & Abrótano hebra & Asteraceae \\
\hline Schkuhria pinnata O. Kuntze & Kills fleas & Asteraceae \\
\hline Senecio jacobaca L. & Ragwort & Asteraceae \\
\hline Tanacetum cinarariae folium & Pyrethrum & Asteráceae \\
\hline Tanacetum vulgare $\mathrm{L}$ & Tansy & Asteraceae \\
\hline Thuja occidentalis L & Tuya & Cupressaceae \\
\hline Thymus vulgaris & Thyme & Lamiaceae \\
\hline Verbena officinalis L. & Verbena & Verbenaceae \\
\hline
\end{tabular}




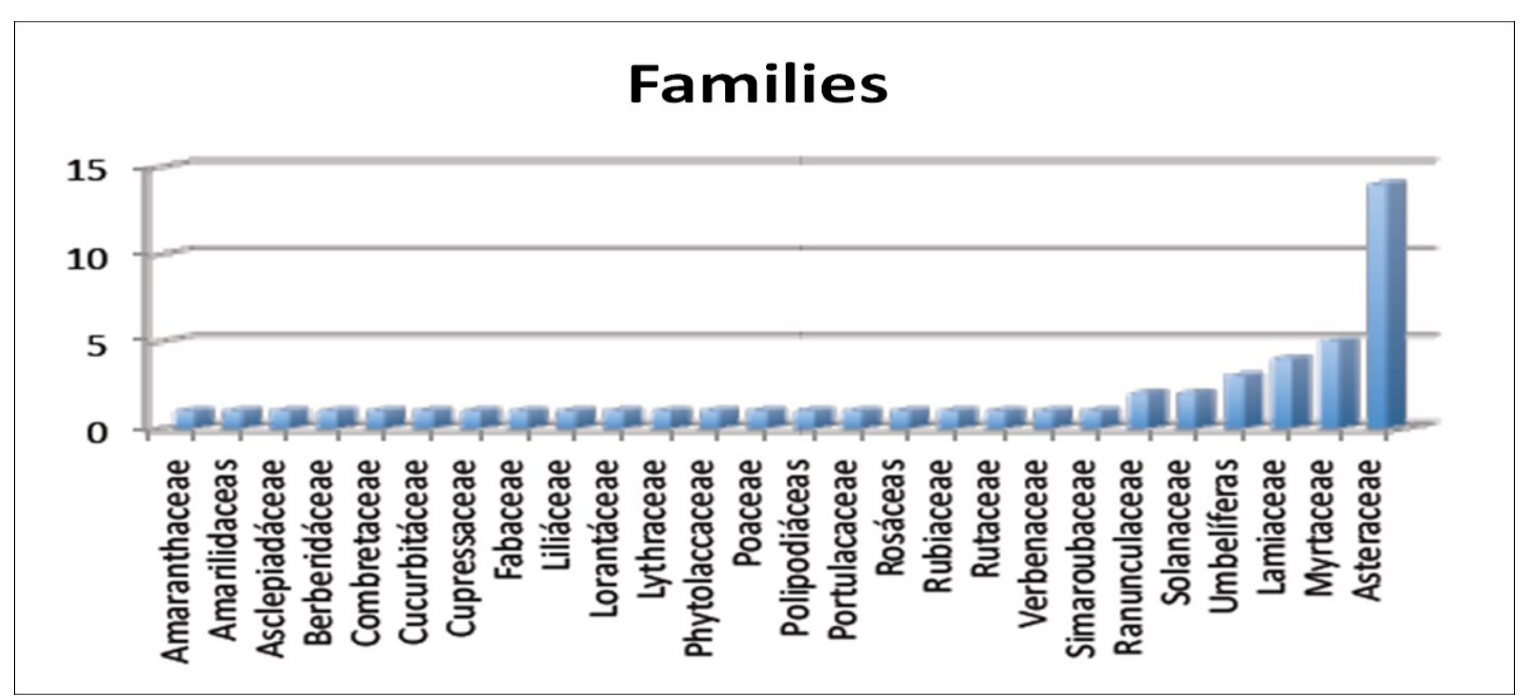

Figure 1. Species according to their families

Table 2 shows a complete data about authors who support its antiparasitic use. It is also been detailed, the part of the plant used, its official use, if the herbs are classified as toxic, and their active ingredients and their possible dosage.

Although the plants are now growing in the Argentine territory, it was explored the original source of them, and this data is shown in figure 2.

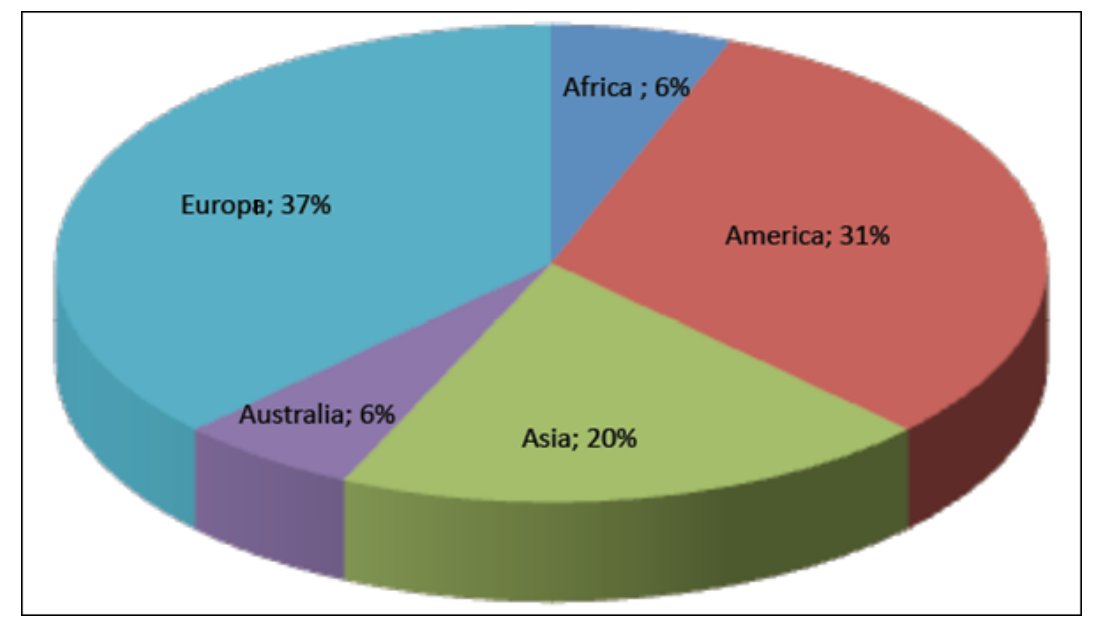

Figure 2. Original source of Argentinean Plants 
Table 2 Indication, Composition and way of use

\begin{tabular}{|c|c|c|c|c|c|c|c|c|}
\hline $\begin{array}{l}\text { Scientific } \\
\text { name/ } \\
\text { Reference }\end{array}$ & $F A$ & $\begin{array}{l}\text { Part } \\
\text { Tox }\end{array}$ & $\begin{array}{l}\text { Part } \\
\text { used }\end{array}$ & Origin & Co $\mathrm{C}$ & Indication & Composition & Way of use \\
\hline $\begin{array}{l}\text { Allium } \\
\text { sativum } L . \\
(6,9,11,12)\end{array}$ & Of & $\mathrm{PE}$ & B & Asc & 1 & $\begin{array}{l}\text { Intestinal antiparasitic } \\
\text { (Oxyuriasis); worming } \\
\text { (amebicida, y } \\
\text { antihelmíntico). } \\
\text { Intestinal } \\
\text { Entamoebahistolytica, } \\
\text { haemonchus contortus; } \\
\text { Giardiaintestina-lis, } \\
\text { Giardialamblia } \\
\text { (Giardiaduodenalis), } \\
\text { Leishmaniamajor, } \\
\text { Leptomonascolosoma, } \\
\text { Crithidiafasciculata, } \\
\text { cryptosporidium baileyi, } \\
\text { Tetratrichomonasgallinar } \\
\text { um, Histomona } \\
\text { smeleagridis } \\
\text { Plasmodium berghei } \\
\text { Trypanosoma brucei } \\
\text { brucei, } \\
\text { T. brucei rhodesiense, } \\
\text { T. brucei gambiense, } \\
\text { T. brucei congolense, } \\
\text { T. evansi, T. equiperdum } \\
\text { T. cruzi }\end{array}$ & Allicin and allyl acid & $\begin{array}{l}\text { Ingestion of raw } \\
\text { bulbs (cooked } \\
\text { loses more than } \\
90 \% \text { effective); } \\
\text { along the high } \\
\text { lemon or mixed } \\
\text { with milk. Very } \\
\text { effective against } \\
\text { the treatment of } \\
\text { worms }\end{array}$ \\
\hline $\begin{array}{l}\text { Artemisina } \\
\text { abrotamum } \\
\text { L. }(7,12)\end{array}$ & Of & PA & $\mathrm{H}, \mathrm{SF}$ & $\mathrm{Em}$ & $1-2$ & $\begin{array}{l}\text { Anti-helmintic, } \\
\text { mosquito repellent }\end{array}$ & $\begin{array}{l}\text { epoxyocimene and } \\
\text { chrysanthenyl acetate }\end{array}$ & $\begin{array}{l}\text { oils obtained by } \\
\text { hydrodistillation } \\
\text { of aerial parts }\end{array}$ \\
\hline $\begin{array}{l}\text { Artemisia } \\
\text { absintium } \\
(11,12,16,21)\end{array}$ & Tox & PE & $\mathrm{H} ; \mathrm{SF}$ & $\mathrm{E}$ & $1-2$ & $\begin{array}{l}\text { Malaria, insect repellent, } \\
\text { pesticide. Worming } \\
\text { (expels parasitic) for } \\
\text { worms and intestinal } \\
\text { worms. }\end{array}$ & $\begin{array}{l}\text { Artemetin } \\
\text { (antimalarial), Beta- } \\
\text { tujon, (Insect repellent, } \\
\text { pesticide). }\end{array}$ & $\begin{array}{l}\text { Infusion, } 2 \mathrm{~g} / \mathrm{L} \text {; } \\
\text { children and } \\
\text { adults, } 10 \mathrm{~g} / \mathrm{L} \text {. } \\
300 \text { a } 400 \mathrm{ml} \text { per } \\
\text { day divided into } 3 \\
\text { doses. }\end{array}$ \\
\hline $\begin{array}{l}\text { Artemisia } \\
\text { maritima }(7)\end{array}$ & Of & PA & $\mathrm{SF}$ & $\mathrm{E}$ & 1 & $\begin{array}{l}\text { Oxyuriasis and } \\
\text { Ascaricida }\end{array}$ & $\begin{array}{l}\text { camphor and 1,8- } \\
\text { cineole }\end{array}$ & $\begin{array}{l}\text { Infusions of } \\
\text { leaves and } \\
\text { flowering tops }\end{array}$ \\
\hline $\begin{array}{l}\text { Artemisia } \\
\text { mendozana } \\
\text { D.C (21) } \\
\end{array}$ & Of & - & $\mathrm{H} \mathrm{SF}$ & A & $1-2$ & $\begin{array}{l}\text { Intestinal antiparasitic } \\
\text { and worming }\end{array}$ & $\begin{array}{l}\text { camphor, artemiseole, } \\
\text { artemisia alcohol and } \\
\text { borneol }\end{array}$ & Infusion $(15 \mathrm{~g} / \mathrm{L})$ \\
\hline $\begin{array}{l}\text { Artemisa } \\
\text { vulgaris, } A \text {. } \\
\text { herba alba } \\
(22,24)\end{array}$ & Of & - & $\mathrm{PE}$ & E As & $1-2$ & Intestinal antiparasitic & $\begin{array}{l}\text { Terpenes and } \\
\text { derivatives 1,8 cineol, } \\
\text { camphor, linalool, } \\
\text { thujone, 4-terpineol, } \\
\text { borneol, cardinol, and } \\
\text { sesquiterpenes }\end{array}$ & $\begin{array}{l}\text { Infusion }(30 \mathrm{~g} / \mathrm{L} \\
\text { of hole plant) }\end{array}$ \\
\hline $\begin{array}{l}\text { Asclepia } \\
\text { curassavica } \\
(25)\end{array}$ & Tox & PE & $\mathrm{R}, \mathrm{T} ; \mathrm{H}$ & A & 1 & $\begin{array}{l}\text { Antiparasitic, } \\
\text { insecticidal and } \\
\text { anthelmintic. }\end{array}$ & $\begin{array}{l}\text { Digital (toxic glucoside) } \\
\text { asclepiadea and } \\
\text { vicentoxina }\end{array}$ & $\begin{array}{l}\text { Infusion of leaves } \\
\text { (antiparasitic), } \\
\text { diluted latex } \\
\text { (insecticide and } \\
\text { anthelmintic) } \\
\end{array}$ \\
\hline $\begin{array}{l}\text { Atractylis } \\
\text { gummifera L } \\
\text { (27) }\end{array}$ & Tox & $\mathrm{R}$ & $\mathrm{PE}$ & $\mathrm{E}$ & $1-2$ & Repellent, insecticide & $\begin{array}{l}\text { Glycosides diterpenices: } \\
\text { atractyloside (ATR) y } \\
\text { carboxi-atractiloside }\end{array}$ & Infusion $30 \mathrm{gr} / \mathrm{L}$ \\
\hline $\begin{array}{l}\text { Castela erecta } \\
\text { ssp }(42)\end{array}$ & Of & - & A & A & $1-2$ & $\begin{array}{l}\text { Antiparasitic effective in } \\
\text { adult parasite and its } \\
\text { status as a cyst in } \\
\text { intestinal mucosa }\end{array}$ & $\begin{array}{l}\alpha \text {-terpinene, camphor, } \\
\text { trans-ascaridol, p- } \\
\text { cymene, pinocarvone, } \\
\alpha \text {-pinene }(3.57 \%) \text { and } \\
\text { geraniol }\end{array}$ & Oil 6 gr $/ \mathrm{L}$ \\
\hline $\begin{array}{l}\text { Chenopodium } \\
\text { ambrosioide } \\
(7,29,47)\end{array}$ & Tox & $\mathrm{PE}$ & $\mathrm{H}, \mathrm{F}$ & A & 1 & $\begin{array}{l}\text { Internal, anthelmintic, } \\
\text { antiparasitic } \\
\text { hookworms, } \\
\text { roundworms, and } \\
\text { oxyrusvermicularis } \\
\text { ascarislumbricoides. } \\
\text { External use as } \\
\text { antisárnica, antiparasitic } \\
\text { and flea scalp. }\end{array}$ & $\begin{array}{l}\text { (Z)-ascaridole, 2-carene, } \\
\rho \text {-cymene, isoascaridole } \\
\text { and } \alpha \text {-terpinene }\end{array}$ & $\begin{array}{l}\text { Decoction not } \\
\text { more than } 5 \mathrm{~g} \text { per } \\
\text { day; for } 3 \text { days } \\
\text { (internal parasite), } \\
\text { external use } \\
\text { (antisárnica and } \\
\text { antiparasitic } \\
\text { scalp); in soils as } \\
\text { flea }\end{array}$ \\
\hline
\end{tabular}




\begin{tabular}{|c|c|c|c|c|c|c|c|c|}
\hline $\begin{array}{l}\text { Cinchona sp } \\
(7,23,32)\end{array}$ & Of & - & $\mathrm{R}$ & $\mathrm{A}$ & 1 & $\begin{array}{l}\text { Antihelmintic, malaria. } \\
\text { In veterinary medicine, } \\
\text { as anti-louse. }\end{array}$ & quinine & Infusion $30 \mathrm{gr} / \mathrm{L}$ \\
\hline $\begin{array}{l}\text { Cuminum } \\
\text { cyminum } L . \\
(7,12)\end{array}$ & Of & - & $\mathrm{F}$ & AfAsm & 1 & Antihelmintic & $\begin{array}{l}\text { Cuminal, cuminic } \\
\text { alcohol, } \gamma \text {-terpinene, } \\
\text { safranal, } p \text {-cymene and } \\
\beta \text {-pinene }\end{array}$ & Infusion $2 \%$ \\
\hline $\begin{array}{l}\text { Curcubita } \\
\text { pepo } L \text {. } \\
(7,11,18,21)\end{array}$ & Of & - & $\mathrm{S}$ & A & $1-2$ & $\begin{array}{l}\text { Intestinal anthelmintic, } \\
\text { Taeniaspp, } \\
\text { Hymenolepisspp, } \\
\text { enterobiusspp, Áscaris, } \\
\text { spp. }\end{array}$ & $\begin{array}{l}\text { Curcubitine, ac. } \\
\text { Linoleic y acoleic, } \\
\text { carotenoides, vit E. }\end{array}$ & $\begin{array}{l}-100 \mathrm{~g} \text { Seeds in } \\
\text { milk -cucurbitita } \\
\text { fasting. } \\
-60 \mathrm{G} \text { of crushed } \\
\text { seeds, } 40 \mathrm{~g} \text { sugar, } \\
60 \mathrm{~mL} \text { of water; } \\
\text { boil for } 20 \mathrm{~min} \text {, } \\
\text { mix without strain. } \\
1 \text { x day followed } \\
\text { purgative }\end{array}$ \\
\hline $\begin{array}{l}\text { Daucus carota } \\
\text { (21) }\end{array}$ & Of & - & $\mathrm{R}$ & Asc & $1-2$ & Antihelmintic & $\begin{array}{l}\text { Carotol, daucene, } \\
\text { farnesene, germacrene }\end{array}$ & $\begin{array}{l}\text { The root raw, } \\
\text { grated, at the rate } \\
\text { of half a kilo to a } \\
\text { kilo for } 24 \text { hours. } \\
\text { At this time } \\
\text { should not eat any } \\
\text { other food. Failing } \\
\text { that, you can take } \\
\text { two carrots daily } \\
\text { fasting for a week. }\end{array}$ \\
\hline $\begin{array}{l}\text { Delphinium } \\
\text { staphisagria } \\
\text { L. }(7,11,34)\end{array}$ & Tox & $\mathrm{PE}$ & $\mathrm{S}$ & $\mathrm{E}$ & $1-2$ & $\begin{array}{l}\text { Antiparasitic Chagas } \\
\text { (flavonoids); pediculosis } \\
\text { (alkaloid is curarizing), } \\
\text { external parasiticide } \\
\text { (toxic), as external } \\
\text { scabies and lice }\end{array}$ & $\begin{array}{l}\text { Alcaloides; metil- } \\
\text { licaconitina }\end{array}$ & $\begin{array}{l}\text { Decoctions seeds, } \\
\text { external } \\
\text { parasiticide }\end{array}$ \\
\hline $\begin{array}{l}\text { Eucalyptus } \\
\text { globulus } \\
(7,18)\end{array}$ & Of & - & $\mathrm{H}-\mathrm{A}$ & $\mathrm{Au}$ & 1 & Anthelmintic & $\begin{array}{l}\text { Eucaliptol y alfa } \\
\text { pineno, ácido caféico y } \\
\text { gálico }\end{array}$ & Tincture \\
\hline $\begin{array}{l}\text { Eugenia } \\
\text { caryophyllus } \\
\text { (12) }\end{array}$ & Of & - & SF & As & $1-2$ & $\begin{array}{l}\text { Acaricidal and } \\
\text { anthelmintic low power }\end{array}$ & eugenol & $\begin{array}{l}\text { Dried flowers } 4 \mathrm{~g} \\
\text { / } 250 \mathrm{ml} \text { of } \\
\text { boiling water; } 15 \\
\mathrm{ml} \text { per day per } 11 \\
\mathrm{~kg} \text { of body weight } \\
\text { for } 2-3 \text { days; } \\
\text { soaked for } 10-20 \\
\text { min. }\end{array}$ \\
\hline $\begin{array}{l}\text { Eugenia } \\
\text { uniflora } \\
(40,41)\end{array}$ & Of & - & $\mathrm{PE}$ & $\mathrm{A}$ & 1 & Antihelmintic & $\begin{array}{l}\text { Lycopene, terpene } \\
\text { compounds, oxalic acid, } \\
\text { chlorogenic acid, } \\
\text { pitagina, cineole, beta } \\
\text { carotene, citric acid. }\end{array}$ & $20 \mathrm{~g} / \mathrm{L}$ of water \\
\hline $\begin{array}{l}\text { Eupatorium } \\
\text { cannabinum L } \\
\text { (7) }\end{array}$ & Tox & $P E$ & $\mathrm{PE}$ & Em & $1-2$ & Antihelmintic, diuretic & $\begin{array}{l}\text { Lactonassequiterpénicas } \\
\text { (eupatoriopicrinay } \\
\text { alcaloids (equinatina, } \\
\text { licopsamine, } \\
\text { intermedine, rinderine y } \\
\text { supinine. }\end{array}$ & $\begin{array}{l}5 \mathrm{~g} \text { of leaves } / 300 \\
\mathrm{ml} \text { of water }\end{array}$ \\
\hline $\begin{array}{l}\text { Hydrastis } \\
\text { canadensis (7) }\end{array}$ & Tox & $\mathrm{R}$ & $\mathrm{H}$ & $\mathrm{A}$ & 1 & Antiparasitic & hidrastina y laberberina & $5 \mathrm{~g} / 100 \mathrm{ml}$ \\
\hline $\begin{array}{l}\text { Inula helenium } \\
\text { L. }(7,8)\end{array}$ & Of & - & $\mathrm{R}$ & Ac & $1-2$ & Intestinal antiparasitic & $\begin{array}{l}\text { Sesquiterpene, } \\
\text { triterpene alcohols, } \\
\text { inulin, helenina, } \\
\text { azulene, sterols, } \\
\text { fructans and mucilage, } \\
\text { essential oil (camphor } \\
\text { énula) }\end{array}$ & $\begin{array}{l}\text { Roots and } \\
\text { rhizomes. } \\
\text { Decoction, } \\
\text { extracts, ointment, } \\
\text { powdered drug, } \\
\text { essential oil } \\
\text { obtained by } \\
\text { distillation. Dry } \\
\text { extract (5: 1) 300- } \\
900 \mathrm{mg} / \text { day }\end{array}$ \\
\hline $\begin{array}{l}\text { Juglans regia } \\
(7,11,12,21)\end{array}$ & Of & - & $\mathrm{H}$ & $\mathrm{Ac}$ & 1 & $\begin{array}{l}\text { Insect repellent; } \\
\text { anthelmintic against } \\
\text { Haemonchuscontortus }\end{array}$ & $\begin{array}{l}\text { Tannins (gallic and } \\
\text { catéquinos): } \\
\text { naphthoquinones } \\
\text { (junglona) }\end{array}$ & $\begin{array}{l}\text { Decoction of } 70 \mathrm{~g} \\
\text { in } 1000 \mathrm{cc} \text { of } \\
\text { water, against the } \\
\text { lonely and to } \\
\text { combat chilblains. } \\
\text { Environmental / }\end{array}$ \\
\hline
\end{tabular}


Vol. 5

\begin{tabular}{|c|c|c|c|c|c|c|c|c|}
\hline $\begin{array}{l}\text { Matricaria } \\
\text { chamomilla } \\
(32,40)\end{array}$ & Of & - & SF & $\mathrm{E}$ & $1-2$ & Antihelmintic & $\begin{array}{l}\text { Flavonoids, apigenin } \\
\text { and luteolin, free and as } \\
\text { heteroside }\end{array}$ & $5 \mathrm{~g} / 100 \mathrm{ml}$ \\
\hline $\begin{array}{l}\text { Melaleuca } \\
\text { leucadendron } \\
\text { (7) }\end{array}$ & Of & - & $\mathrm{H}$ & $\mathrm{Au}$ & $1-2$ & Scabies and pediculosis & $\begin{array}{l}\text { 1,8-cineol, nerolidol, } \\
\text { alloaromadendrene y } \alpha \text { - } \\
\text { terpineol }\end{array}$ & $\begin{array}{l}\text { Environmental / } \\
\text { repellent }\end{array}$ \\
\hline $\begin{array}{l}\text { Mentha } \\
\text { piperita } \\
(12,32)\end{array}$ & Of & - & H SF & Em & 1 & $\begin{array}{l}\text { Ectoparasiticidal, } \\
\text { helminths (worms and } \\
\text { other antiparasitic } \\
\text { digestive), worming. }\end{array}$ & $\begin{array}{l}\text { Essential oil (menthol, } \\
\text { menthone, methyl } \\
\text { acetate, mentafurano, } \\
\text { isomenthone, pulegone, } \\
\text { piperitone, cineol, } \\
\text { limonene), flavonoids } \\
\text { (apigenol, luteol, } \\
\text { rutoside, mentósido) } \\
\text { ácidosfenólicos (caffeic, } \\
\text { chlorogenic, } \\
\text { rosmarinic), tannins, } \\
\text { triterpenes } \\
\text { (ácidoursólico, } \\
\text { ácidooleanílico) }\end{array}$ & $\begin{array}{l}\text { Environmental / } \\
\text { repellent }\end{array}$ \\
\hline $\begin{array}{l}\text { Mentha } \\
\text { pulegium L. } \\
\text { (7) }\end{array}$ & Tox & $\mathrm{H}, \mathrm{A}$ & SF, H & $\mathrm{E}$ & 1 & insect repellent & $\begin{array}{l}\text { Essential oil (pulegone, } \\
\text { 1-menthone, d- } \\
\text { isomenthone, } \\
\text { piperitone, piperitenone, } \\
\text { isopiperitenona), } \\
\text { alcohols (menthol, 3- } \\
\text { octanol,), esters } \\
\text { (menthyl acetate), } \\
\text { hydrocarbons (alpha } \\
\text { and beta-pinene, } \\
\text { limonene, p-cymene, } \\
\text { dipentene, camphene) }\end{array}$ & $\begin{array}{l}\text { Essential oil -1- } \\
2 \text { gts / 12hs- } \\
\text { Infusion 1cda / } \\
\text { cup; take 2-3tazas } \\
\text { / day; } \\
\text { Environmental / } \\
\text { do bunches, } \\
\text { repellent }\end{array}$ \\
\hline $\begin{array}{l}\text { Mentha } \\
\text { spicata (32) }\end{array}$ & Of & - & $\mathrm{H}$ & $\mathrm{E}$ & 1 & worming & $\begin{array}{l}\text { alpha-pinene, limonene, } \\
\text { 1-carvone and ketone. }\end{array}$ & oral \\
\hline $\begin{array}{l}\text { Myroxylon } \\
\text { balsamiferum } \\
\text { (7) }\end{array}$ & Of & - & SF & A & 1 & scabies, lice, mites & $\begin{array}{l}\text { Cinnameine, benzoate, } \\
\text { cinamate benzile, } \\
\text { nerolirol \& peruviol }\end{array}$ & $\begin{array}{l}15 \mathrm{~g} \text { with } 19 \mathrm{~g} \text { of } \\
\text { ricine oil and } \\
75 \mathrm{gr} \text { vaseline } \\
\text { (external use) }\end{array}$ \\
\hline $\begin{array}{l}\text { Nephrodium } \\
\text { (11) }\end{array}$ & Of & - & $\mathrm{R}$ & A & $1-2$ & Anthelmintic, tapeworm. & $\begin{array}{l}\text { quinoid, albaspidine, } \\
\text { tris-para-aspidine, } \\
\text { philíxic acid, } \\
\text { flavaspidic, tetra- } \\
\text { flavaspidic, aspidinole, } \\
\text { filicine, filicilic acid \& } \\
\text { flavaspidic acid }\end{array}$ & $\begin{array}{l}\text { Manage capsule } \\
\text { ether extract of } \\
\text { male fern, } \\
\text { administered one } \\
\text { capsule every } 10 \\
\text { minutes and one } \\
\text { hour after taking a } \\
\text { saline cathartic, } \\
\text { never oily. }\end{array}$ \\
\hline $\begin{array}{l}\text { Nicotiana } \\
\text { glauca } \\
\text { Graham } \\
(42,44)\end{array}$ & Of & - & $\mathrm{H}$ & A & $1-2$ & $\begin{array}{l}\text { Ectoparasiticidal and } \\
\text { anthelmintic }\end{array}$ & $\begin{array}{l}\text { Anabasine, pireridina, } \\
\text { nicotine, nornicotine, } \\
\text { citric, succinic, malic } \\
\text { and oxalic acid and } \\
\text { rutin. }\end{array}$ & brews; toxic \\
\hline $\begin{array}{l}\text { Nicotiana } \\
\text { tabacum } \\
(12,46,47,48)\end{array}$ & Tox & $\mathrm{PE}$ & $\mathrm{H}$ & $\bar{A}$ & $1-2$ & $\begin{array}{l}\text { Scabies; leeches, } \\
\text { ectoparasiticidal and } \\
\text { helminths. }\end{array}$ & $\begin{array}{l}\text { Nicotine; Anabasine, } \\
\text { piperidine nicotine, } \\
\text { nornicotine and N- } \\
\text { methyl anabasine }\end{array}$ & $\begin{array}{l}\text { Dust; 0,2-2g / } \\
\text { day divided into } 3 \\
\text { doses; Infusion } \\
\text { 1cda / 3tazas / } \\
\text { day; 1-2gts } \\
\text { essential oil / 8- } \\
\text { 24hs (no more } \\
\text { than 5gts per } \\
\text { sample) }\end{array}$ \\
\hline $\begin{array}{l}\text { Petiveria } \\
\text { alliacea } \\
(24)\end{array}$ & Of & - & $\mathrm{R}, \mathrm{T}$ & A & 1 & Scabies, worming & $\begin{array}{l}\text { benzaldehyde, benzoic } \\
\text { acid, benzyl 2- } \\
\text { hydroxyethyl } \\
\text { trisulphide,coumarin, } \\
\text { isoarborinol, } \\
\text { isoarborinol acetate, } \\
\text { isoarborinol, } \\
\text { cinnamate, } \\
\text { isothiocyanates, } \\
\text { polyphenols, senfol, } \\
\text { tannins, trithiolaniacin }\end{array}$ & $\begin{array}{l}30 \mathrm{~g} \text { of roots in } \\
\text { one litre of water }\end{array}$ \\
\hline
\end{tabular}




\begin{tabular}{|c|c|c|c|c|c|c|c|c|}
\hline $\begin{array}{l}\text { Pimpinella } \\
\text { anisum L. (7) }\end{array}$ & Of & - & $\mathrm{F}$ & A & 1 & Anti-worms & $\begin{array}{l}\text { Essential oil (t-anethole, } \\
\text { estragole, } \\
\text { anisaldehyde), } \\
\text { furanocumarinas } \\
\text { (umbelliferone), terpene } \\
\text { hydrocarbons, } \\
\text { stigmasterol, flavonoids } \\
\text { (quercitrósido, } \\
\text { isoorientin, vitexin, } \\
\text { rutin), choline, malic } \\
\text { acid }\end{array}$ & $\begin{array}{l}\text { Infusion } 1 \\
\text { tablespoon / } 3 \\
\text { cups / of; 1-2gts } \\
\text { essential oil / 8- } \\
\text { 24hs (no more } \\
\text { than 5gts per } \\
\text { sample) }\end{array}$ \\
\hline $\begin{array}{l}\text { Podophyllum- } \\
\text { peltatum } L . \\
(7,49)\end{array}$ & Tox & $\mathrm{R}$ & $\mathrm{H} ; \mathrm{T} ; \mathrm{R}$ & A & $1-2$ & $\begin{array}{l}\text { Anthelmintic (anti- } \\
\text { worm) }\end{array}$ & $\begin{array}{l}\text { Podophyllum resin } \\
\text { (lignans } \\
\text { [podophyllotoxin } \\
\text { picropodophyllin, } \\
\text { peltatin alpha, beta- } \\
\text { peltatin] } \\
\text { heterósidosglucosides) }\end{array}$ & $\begin{array}{l}\text { Decoction of the } \\
\text { root bark as } \\
\text { tenífugo, } \\
\text { accompanied by a } \\
\text { purgative to cause } \\
\text { the expulsion of } \\
\text { tapeworm }\end{array}$ \\
\hline $\begin{array}{l}\text { Portulaca } \\
\text { oleracea } L . \\
(7,28)\end{array}$ & Of & - & sumo & As & 1 & anthelmintic & $\begin{array}{l}\text { oleraceínas A, B, C, D y } \\
\text { E; diterpenoide } \\
\text { portulene, lupeol, } \\
\text { betasitosterol y } \\
\text { daucosterol }\end{array}$ & $\begin{array}{l}2.5 \mathrm{mg} \text { of } \\
\text { leaves } / 100 \mathrm{ml} \\
\text { water, } 80 \mathrm{mg} \text { of } \\
\text { seeds } / 100 \mathrm{ml} .\end{array}$ \\
\hline $\begin{array}{l}\text { Punica } \\
\text { granatum } L \\
(7,11,23)\end{array}$ & Of & - & $\mathrm{R}, \mathrm{T}, \mathrm{F}$ & As & $1-2$ & $\begin{array}{l}\text { Tenias, intestinal } \\
\text { antiparasitic, Vermifuge, } \\
\text { amebicide, tapeworm, } \\
\text { intestinal worms. }\end{array}$ & $\begin{array}{l}\text { Alkaloids, less in the } \\
\text { fruit (peletierina, } \\
\text { isopeletierina, } \\
\text { pseudopeletierina, N- } \\
\text { metilpeletierina) } \\
\text { hydrolysable tannins } \\
(3,6- \\
\text { hexahidrofenoilglucosa, } \\
\text { granatinas A and B, } \\
\text { punicafolina, } \\
\text { punicacorteínas A, B } \\
\text { and C, punigluconina, } \\
\text { punicalin, punicalagin) }\end{array}$ & $\begin{array}{l}\text { worming; } 60 \mathrm{~g} \text { of } \\
\text { root bark or stem / } \\
\text { water } 500 \mathrm{ml} \text { of } \\
\text { macerate for } 24 \\
\text { hours, boiled to } \\
\text { reduce the volume } \\
\text { to half. Take } \\
\text { fasting. After an } \\
\text { hour and a half, } \\
\text { catharsis because } \\
\text { it tends to dry the } \\
\text { belly. }\end{array}$ \\
\hline $\begin{array}{l}\text { Quassia } \\
\operatorname{amara}(29)\end{array}$ & Of & - & $\mathrm{R}, \mathrm{T}$ & A & $1-2$ & $\begin{array}{l}\text { Intestinal worms and } \\
\text { insecticide (mites, } \\
\text { beetles, Hemiptera, } \\
\text { Hymenoptera, } \\
\text { Lepidoptera and } \\
\text { thisanópteros) } \\
\text { Plasmodiumyoelii and P. } \\
\text { falciparum. }\end{array}$ & $\begin{array}{l}\text { quasinoides, } \\
\text { simalikalactone D }\end{array}$ & $\begin{array}{l}\text { Infusion: } 1-4 \mathrm{~g} \\
\text { cup. Tincture: } 25 \\
\text { gts, three times a } \\
\text { day }\end{array}$ \\
\hline $\begin{array}{l}\text { Ruta } \\
\text { chalepensis } \\
(11,50)\end{array}$ & Tox & PA & PA & $\mathrm{E}$ & $1-2$ & intestinal worms & $\begin{array}{l}\text { nonylmethyl ketone; } \\
\text { glucoside routine and } \\
\text { essential oil. Gums, } \\
\text { resins, tannin materials }\end{array}$ & $\begin{array}{l}1 \mathrm{~kg} \text { of whole } \\
\text { plant in } 1 \text { litre of } \\
\text { boiling wáter. }\end{array}$ \\
\hline $\begin{array}{l}\text { Santolina } \\
\text { chamaecy- } \\
\text { parissus (7) }\end{array}$ & Of & - & $\mathrm{T}$ & $\mathrm{E}$ & $1-2$ & $\begin{array}{l}\text { Aphid repellent; } \\
\text { Anthelmintic (to } \\
\text { eliminate intestinal } \\
\text { antiparasitic) of } \\
\text { children. }\end{array}$ & $\begin{array}{l}\text { artemisia ketone, } \\
\text { camphor, and } \beta \text { - } \\
\text { phellandrene }\end{array}$ & $\begin{array}{l}\text { Repellent infusion } \\
\text { leaves }\end{array}$ \\
\hline $\begin{array}{l}\text { Schkuhria } \\
\text { pinnata } O . \\
\text { Kuntze (7) }\end{array}$ & Of & - & $\mathrm{PE}$ & $\mathrm{E}$ & $1-2$ & Insecticide; antimalarial & $\begin{array}{l}\text { sesquiterpene lactones; } \\
\text { heliangolide }\end{array}$ & $\begin{array}{l}15 \mathrm{~g} \text { of leaves in } 1 \\
\text { litre of water }\end{array}$ \\
\hline $\begin{array}{l}\text { Senecio } \\
\text { jacobaca } L . \\
(7,51)\end{array}$ & Tox & $\mathrm{PE}$ & $\mathrm{SF}$ & $\mathrm{E}$ & $1-2$ & Antihelmintic & $\begin{array}{l}\text { Metilheptilcetona, l-a- } \\
\text { pinen, cineol, 1- } \\
\text { limoneno y metil-n- } \\
\text { nonilcarbinol. }\end{array}$ & $\begin{array}{l}\text { internal use. } \\
\text { Infusion } 1 \\
\text { tablespoon / } \\
150 \mathrm{ml}, 3 \text { cups / } \\
\text { day, essential oil } \\
1-5 \text { gts }(25-50 \mathrm{mg}) \\
\text { / } 8 \text { hours after } \\
\text { meals }\end{array}$ \\
\hline $\begin{array}{l}\text { Tanacetum } \\
\text { cinerariae- } \\
\text { folium }(7,32)\end{array}$ & Tox & SF & SF & As & $1-2$ & $\begin{array}{l}\text { Antiparasitic, } \\
\text { insecticidal }\end{array}$ & $\begin{array}{l}\text { essence; esters } \\
\text { (piretrolona, cinerolona } \\
\text { and jasmolona) }\end{array}$ & $\begin{array}{l}\text { alcoholic } \\
\text { solutions, lotions } \\
\text { and shampoos. }\end{array}$ \\
\hline $\begin{array}{l}\text { Tanacetum } \\
\text { vulgare } L \\
(7,40)\end{array}$ & Tox & $\mathrm{SF}$ & $\mathrm{SF}$ & $\mathrm{Eu}$ & $1-2$ & Insecticidal & $\begin{array}{l}\text { 1,8-cineole, trans- } \\
\text { thujone, camphor and } \\
\text { myrtenol }\end{array}$ & $\begin{array}{l}\text { aerial parts in } \\
\text { bundles to insects, } \\
\text { environmental use }\end{array}$ \\
\hline
\end{tabular}




\begin{tabular}{|c|c|c|c|c|c|c|c|c|}
\hline $\begin{array}{l}\text { Thuja } \\
\text { occidentalis L } \\
(7,52) \text {. }\end{array}$ & Tox & PA & $\mathrm{T}$ & $\mathrm{A}$ & 1 & Anthelmintic, insecticide & Alpha-thujone & $\begin{array}{l}20 \mathrm{~g} \text { of leaves in } 1 \\
\text { litre of boiling } \\
\text { water }\end{array}$ \\
\hline $\begin{array}{l}\text { Thymus } \\
\text { vulgaris }(7,53)\end{array}$ & Of & & $\mathrm{H}, \mathrm{SF}$ & E y Af & 1 & intestinal antiparasitic & $\begin{array}{l}\text { Essential oil (thymol, } \\
\text { carvacrol, p-cymene, } \\
\text { beta-pinene, borneol, } \\
\text { linalool, geraniol, } \\
\text { terpineol, t-tuyanol- } \\
\text { terpineol) } \\
\text { ácidostriterpénicos } \\
\text { (urólico, oleanolic), } \\
\text { ácidocafeico, } \\
\text { ácidorosmarínico, } \\
\text { flavonoids (luteolin, } \\
\text { tetrahydroxy-5,7, } 3 \text { ', } \\
\text { 4'-flavone), triterpene } \\
\text { saponins, tannins }\end{array}$ & $\begin{array}{l}\text { Infusion } 5 \% \text { 2-3 } \\
\text { cups a day. } \\
\text { Essential oil; 1-5 } \\
\text { drops per dose, on } \\
\text { a sugar cube or in } \\
\text { aqueous solution }\end{array}$ \\
\hline $\begin{array}{l}\text { Verbena } \\
\text { officinalis } L \text {. } \\
\text { (23) }\end{array}$ & Of & & $\mathrm{H}, \mathrm{T}$ & $\mathrm{E}$ & 1 & Antihelmintic & $\begin{array}{l}\beta \text {-sitosterol, ursolic } \\
\text { acid, oleanolic acid, 3- } \\
\text { epiursolic acid, 3- } \\
\text { epioleanolic acid, } \\
\text { iridoid glucosides } \\
\text { (verbenalin \& } \\
\text { hastatoside), } \\
\text { phenylpropanoid } \\
\text { glycoside, verbascoside } \\
\text { and } \beta \text {-sitosterol-D- } \\
\text { glucoside }\end{array}$ & $\begin{array}{l}\text { Infusion } 25 \mathrm{~g} \text { in } \\
\text { one liter of water }\end{array}$ \\
\hline \multicolumn{9}{|c|}{$\begin{array}{l}\text { FA: As listed in the pharmacopoeia Argentina 8th Ed. Official drug (Of) or Toxic Drugs (Tox). Toxic or part as Official Drug Formulary = R: root, } \\
\text { PE: whole plant; PA: aerial parts; H: leaves; A: oils } \\
\text { Part used = B: bulbs; S: seeds; SF: flowering tops; F: fruit, T: branches and stems. Originally = E: Europe; A: America, Af: Africa; As Asia; Asc } \\
\text { Central Asia; Au: Australia; Em: Mediterranean Europe; } \\
\text { C and / or C: crop growth in Argentina: 1: those species that grow naturally or spontaneously. 2: those that are grown. }\end{array}$} \\
\hline
\end{tabular}

\section{The sources used:}

We have observed an extensive use of plants that have European origins, maybe based in the fact that Argentine population has their roots predominantly in European countries like Italy and Spain. Mediterranean area of these countries has similar climate, temperature and humidity than vast areas of Argentina (figure 2).

The main part used from plants were leaves, stems, whole plant, seeds, aerial parts, flowering tops, fruits, root, oil or juice (Table 2, Figure 3).

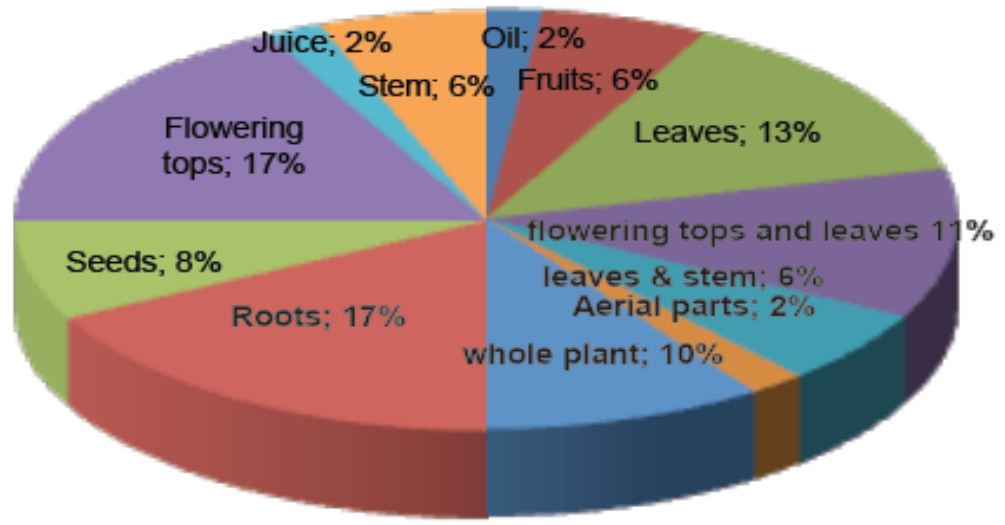

Figure 3. Part of the plant used

\section{Analysis of the most significant plants}

The bulb of Allium sativum L. known as the "Garlic" belonging to the family of a liliáceaes shown to have activity against different parasites [7] intestinal parasites (oxyuriasis) [8-10], or intestinal worms [11]. Its effectiveness is due to the presence of the allylic and acid allicin, which would confer the amebicide and anthelmintic properties.

Experiments with intestinal Entamoeba histolytica parasite have shown that pure allicin inhibits histopathological effects associated with infection and parasite growth; effective against 
Haemonchus contortus in ruminant hosts; diluted cryptosporidium parasites in humans in preliminary tests; inhibits Entamoeba histolytica growth trophozoite in vitro.

Whole garlic extract demonstrated anti-Giardia effectiveness and activity against other microaerophilic flagellate intestinalis [12]. Allicin diallyl trisulfide product (DAT) and ajoene, inhibits the growth of different protozoan parasites, including Giardia lamblia (Giardia duodenalis), Leishmania major, Leptomonas colosoma, Crithidia fasciculata, Cryptosporidium baileyi, Tetratrichomonas gallinarum, Histomonas meleagridis Plasmodium berghei Trypanosoma brucei brucei, T. brucei rhodesiense, T. brucei gambiense, T. brucei congolense, T. evansi, Tequiperdum . and T. cruzi.

Ajoene is effective against T. cruzi epimastigotes and amastigotes. With such trypanocidal activity, garlic derivates could be used as an alternative treatment for African-trypanosomiasis, considering that current drugs for brain infections (sleeping sickness) are extremely toxic.

The mechanism of action of the allicin and ajoene is through its interaction with thiol groups present on enzymes, inhibiting cysteine proteases, alcohol dehydrogenases and thioredoxin reductases of parasites like E. histolytica. Ajoene in the $T$. cruzi inhibits proliferation, possibly by inhibiting the biosynthesis of phosphatidylcholine, by interfering with traffic of ${ }^{\circ}$ proteins and lipids in the cell membranes of the parasite. Ajoene inhibits the final stage of the biosynthesis of phosphatidylcholine and modifies of the composition of the cell membrane phospholipids [13]. Many polyphenols (hydrolysable tannins, proanthocyanidins, caffeic acid products) demonstrated to reduce intracellular survival form of Leishmania donovani amastigotes in vitro. The effects of polyphenols against intracellular parasites of Leishmania are due to the activation of macrophages rather antiparasitic activity $[9,14,15]$.

Within the genus Artemisia, belonging to the family of asteraceaes we identified 5 species with antiparasitic properties including the popularly known since ancient times Artemisia absintium. But it is noteworthy that the similarity between them allows the use of one specie instead of the other.

The leaves and flowering tops of Artemisia absintium commonly known as "Wormwood" in the family of asteraceaes possess a demonstrated antimalarian effect in countless articles. It has Beta-thujone, which gives it a pesticide and insect repellent effect. The sequiterpene lactones, would serve to combat intestinal parasites, but their use is limited because its toxicity. It has been used for centuries as an anthelmintic, especially against roundworms and earthworms [11]. Since the essential oil is volatile, and absintine contains thujone, the product is extremely powerful, so, even at very low doses, it may cause coma or death in adults. For this reason, it is preferred to use dried plant material instead of essential oil $[12,16]$. It should be keep in mind that in the Pharmacopoeia Argentina VIII edition, this plant drug is classified by its toxic properties.

Popularly, wormwood tincture of Artemisia is used for other purposes, such as hallucinogen.

Artemisia contains santonin which has a selective toxic action on the node located in the nerve ring of Ascaris spp. However, it narrow range of activity does not include Oxyuris spp. or tapeworms $[11,17]$.

It is published that the aerial parts of this family of plants are effective against nematodes and worms (20-30 g aerial parts in $500 \mathrm{ml}$ of boiling water for 20 minutes, cool, strain and take $15 \mathrm{ml} /$ $\mathrm{kg}$ body weight). The sweet wormwood (Artemisia annua) has less negative components of $A$. absinthium [12].

On the other hand, the infusion of the leaves and stems has proven its action against amoeba (Entamoeba histolytica) $(2 \mathrm{~g} / \mathrm{L}$, children and adults, $10 \mathrm{~g} / \mathrm{L}$. In all cases take 300 to $400 \mathrm{~mL}$ per day in 3 divided doses). It should be noted that the essential oil contains highly toxic substances foliage, so, not controlled use can cause vomiting, dizziness, seizures, hallucinations, disorders of sensation and other severe diseases of the CNS if doses are exceeded [18].

Annua, used in the Río de la Plata because its antiparasitic properties, is active in wild wormwood. [12, 19]. It is also used in Chinese medicine for its proven activity as antimalaric against Plasmodium falciparum [20]. 
The leaves, flowering tops and essence of Artemisia abrotamum L. popularly known as "Abrotane male", is given a high level of effectiveness and its use as an anthelmintic [6, 7, 12].

Infusions of leaves and flowering tops of Artemisia mendozana are well known because of its vermifuge properties against wormwood for intestinal parasitism treatment [21].

Literature also mentions its use by Mapuche's communities of NW Patagonia. The flowering tops of Artemisia maritima L popularly known as "Wormwood Maritime" has been shown to be active against threadworm and ascaris but its toxicity is similar to castor oil [7] and can produce visual disturbances as side effects.

The oil obtained by distillation of whole plant of $A$. herba albay vulgaris popularly known as "Artemis", is an essential oil that contains plenty different terpenes and terpene derivatives (1.8 cineol, camphor, linalool, tujone, 4-terpineol, borneol, a-Cardinol), artemisia ketone, flavonoids, poliínos, numerous coumarins and sesquiterpene lactones (artemisinin artemisinic acid arteanuina $\mathrm{B}$, etc.). It was used as an anthelmintic, but has fallen into disuse because of its toxicity, adverse reactions such as allergies and abortive activity [20].

Its use as an antimalarial in cases of severe falciparum malaria in situation of resistance to other antimalarials [22].

In conclusion, Artemis has a very old reputation as an anthelmintic [23]. All Artemis species contain thuyona Artemisia essential oil which is somewhat toxic. Because of this, these plants should not been used in high doses, since it can cause dizziness, headache, convulsions, hallucinations, addiction, tremors, nausea and vomiting [21].

The Asclepia popularly known as "Spanish Banderita or Spanish Flag" belongs to the family of Asclepiadáceaes. The whole plant is considered toxic (Ed. FA VIII) and its milky juice is caustic. However it is recorded in ethnomedicine that this specie has anti-parasitic (with leaf infusion), insecticidal and anthelmintic actions (diluted latex). In Aldama, México few drops of milky juice in $500 \mathrm{ml}$ of water are consume throughout the day [24] as anthelmintic and also for cardiac stimulation -since it has glucoside action- $[25,26]$.

The root Atractylis gummifera L popularly known as "League" in the family of Asteraceaes is classified as toxic plant drug (FA VIII Ed.) but records shows that mixed with food acts as a repellent and insecticide for bug and external parasites. The mechanism of action is explained because the composition contains atractyloside (ATR) and carboxiatractiloside, two diterpene heterosides that cause inhibition of the respiratory chain at the cellular level (C Hamouda, 2004). The toxic effect arises from ATR, a potent inhibitor of oxidative phosphorylation in mitochondria [27].

The essence oil Castile erecta ssp popularly known as "Bizbirinda", "bitter squat" belonging to the family of Lorantáceaes destroys parasites in adulthood and its cyst form in the intestinal mucosa [28].

The leaves and fruit of Chenopodium ambrosioides L. popularly known as "Goosefoot", "Piako", "paico", belonging to the family of Amaranthaceaes, is considered vermifuge since the oil obtained from the whole plant is toxic for parasites (FA VIII Ed.) Chenopodium oil is known as an effective anthelmintic due to its contents of ascaridol. Although it is widely used, however, the application is not easy since it must be followed by a treatment with a laxative, immediately after ingestion.

Infusion and decoction for internal is used as an external antiparasitic and anthelmintic and it is also use in topical applications as antiparasitic and scabicide. Dry powder and seeds are used against parasites, hookworms and tapeworms, and on the floor like flea [28].

In a rural community in Huaraz, Peru, a clinical essay with 60 children, aged 3 to 14 years old, was performed dividing these patients in a randomized way into two groups of treatment Albendazole and Paico ( $\mathrm{n}=30$ each group). All the patients were initially evaluated as positively for Áscaris lumbricoides in stool. Treatment consisted of Paico juice: $1 \mathrm{ml} / \mathrm{kg}$ for children less than $10 \mathrm{~kg}, 2 \mathrm{ml} / \mathrm{kg}$ dose for 3 consecutive days. Administration of Albendazole was a done by a single dose of $400 \mathrm{mg}$ in children over 5 years and $200 \mathrm{mg}$ in young adults. The main objective 
parameter was the complete disappearance of Ascaris eggs and additional quantitative parameter was reduced parasite load.

The research was performed in the laboratory of the Regional Health Directorate of Ancash. Eradication rates for Ascaris with Albendazole or Paico were both about $86 \%$. Quantitative effectiveness was 59.5\% and 58.3\% for Paico and Albendazole respectively. The authors conclude that Paico have similar efficacy that Albendazole against Ascaris lumbricoides. Paico also had the added benefit of being effective against Hymenolepis nana [29].

Another study of leaf extracts in two villages near Tarapoto, San Martin, Peru included 72 patients with intestinal parasitic infections. Status was examined before and after 8 days after ingestion, confirming $100 \%$ of the efficacy against Ancylostoma and Trichuris and 50\% against Ascaris. There were no significant differences in age or sex [30].

The root bark and trunk of Cinchona spp, popularly known as "Quina" belonging to the family of Rubiaceaes, has antimalarial activity, since it is rich in phenolic compounds including you cinconaines; proantocianidoles, quinic acid, saponins, alpha-terpineol, linalool, limonene , terpenes [31,32]. Anti-malarial activity of quinine was demonstrated either for treatment and prophylaxis of Plasmodium vivax malarias, $P$. falciparum, $P$. ovale and P. malarie and inactivation of sporozoites and tissue forms [23].

The leaves and stems of Combretum micranthum L popularly known as "Combretum" belonging to the family of Combretaceaes; contains alkaloids, betaine derivatives, as staquidrine, 4hydroxiestaquidrine, combretine-A; flavones as vitexin, saponaretine, orientin and tannins catechic. Activity against parasite referred to its alkaloids compounds but results are limited [7,33].

Seeds of Cucurbita popularly known as "Pumpkin" belonging to the family of Cucurbitáceae is active against Taenia spp, Hymenolepis spp, Enterobius spp, Ascaris spp, [11, 18, 21]. This action is due to the cucurbitine which is active in trematodes, but inactive against nematodes and cestodos. The advantage this family is that it has no hepatic and gastrointestinal toxicity [32]. Formulation could be prepare as follows: $60 \mathrm{~g}$ of crushed seeds, $40 \mathrm{~g}$ of sugar, $60 \mathrm{~mL}$ of water, boiled for $20 \mathrm{~min}$, mixing and emulsifying unstrained. Patients should take 1 portion daily [18].

The seeds of Delphinium staphisagria L. popularly known as "Estafisgria", or "Kill lice", belonging to the family of Ranunculaceaes, demonstrated antiparasitic action, specially active against pediculosis, scabies and Chagas disease [7,34]. Since it contains (toxic) alkaloids, it is recommend to use is as external plant powder as environment repellent.

From dried flower of Eugenia caryophyllus popularly known as "Nail" belonging to the family of Myrtaceaes, it can be obtained an essential oil that showed acaricidal activity against Dermatophagoides farinae and D. pteronyssinus eugenol, Psoroptes cuniculi. Dried flowers ground cloves (Eugenia caryophyllus) are also prepared as a tea infusion with $4 \mathrm{~g}$ of powdered herb per 250 $\mathrm{ml}$ of boiling water. This should be soaked for 10-20 min, cooled and strained. The dose used was $15 \mathrm{ml}$ per day per $11 \mathrm{~kg}$ of body weight for 2-3 days. Oil from this plant is not considered safe for pets [12]. Eugenia caryophyllus may contribute to parasitide action either alone or in combination. Alpha-thujone, the active ingredient of wormwood oil, was reported with insecticide and antihelmintic activity [35]. The eugenol and caryophyllene in Eugenia caryophyllata are also informed as anti-helmintic compounds [36,37,38].

The whole plant of Eugenia uniflora popularly known as "ñangapiry pitanga " belongs to the family of Myrtaceaes, and is found in the tropical jungles of Argentina, Brazil, Paraguay, southern Mexico and Paraguay. It is used in the Guarani communities for their antihelmintic properties [39,40,41]. The plant contains licopene composition, terpene compounds, oxalic acid, chlorogenic acid, pitagina, cineol, beta carotenesand citric acid [41,42].

The root of Inula helenium L. rhizome popularly known as "Elecampane, grass moro" belongs to the family of Asteraceaes and has an intestinal antiparasitic properties. Sequiterpenos found in its composition, triterpene alcohols, inulin, helenina, azulene, sterols, fructans and mucilages, essential oil (Enula camphor) [7,43] are the basis of its vermifuge action. Decoction of the roots and 
rhizomes, ointment extracts, powdered drug and essential oil obtained by distillation are all formulations used in traditional medicine.

The dried leaves of Juglans regia popularly known as "Walnut" belonging to the family of Asteraceaes, originally from Persia and cultivated in North-West of Argentina have demonstrated anthelmintic activity against Haemonchus contortus in goats. Juglone, and naphthoquinone are the compounds of this family reported with anthelmintic activity $[7,11,12,32]$. It exist research data demonstrating that decoction and crushing and soaking water of this plant is useful as insect repellent [21].

The flowering tops Matricaria chamomilla popularly known as "Manzanilla" belongs to the family of Asteraceaes. In infusion the plant only released 10 to $15 \%$ of the total content of essential oils. This oil is mainly composed of sesquiterpenes (azulene), and bisaboloides (camazulenbisabolol). On the other hand, water-soluble portion contains flavonoiglicosides, terpene, pectins and various oligosaccharides compounds.

It is considered as FA official drug, but its indication does not refer to the antiparasitic property, however, in Argentina it is use as an anti-helmintic agent. Some authors refer their activity mainly due to its flavonoids (apigenin and luteolin). [32,40].

The essential oil obtained from the leaves and dried flowering tops of Mentha piperita popularly known as "Mint" belongs to the family of Lamiaceaes. It has the property to remove worms and other parasites from the digestive tract, hence the Spanish proverb "jury is mint, never lie to the stomach" is based in this herb's action. Also is used for environmental repellent in ways bunches. The leaves contain essential oil (menthol, menthone, methyl acetate, mentafurane, isomenthone, pulegone, piperitone, cineol, limonene), flavonoids (apigenol, luteol, rutoside, mentosido), phenolic acids (caffeic, chlorogenic, rosmarinic), tannins and triterpenes (ursolic acid, oleanilic acid) [12,32]. Mentha cordifolia contains beta-sitosterol glycosides and has been tested against Ascaris suum showing a similar synthetic anthelmintic mebendazole on contact with the preparations of the parasite activity. Mint spp., has also been tested with succeed against Haemonchus contortus in ruminant hosts [12].

Balsamum Myroxylon popularly known as "Balm of Peru" belonging to the family Fabaceae is considered by Drugs Official USP as parasiticide and anthelmintic drug. Its resin is used to mange, lice, mites [7].

The root of Nephrodium popularly known as "male fern" belonging to the family of Polypodiaceae, is used for treatment against tapeworm. It is suggested to take either 1 capsule or drink an extract every 10 minute one hour after taking a saline purgative. It is not recommended in children, elderly, or patients suffering from kidney's disease [11].

Leaves of Nicotiana glauca Graham popularly known as "Palán-Palán" belonging to the family Solanaceae, is well known for its toxicity property due to anabasine, an alkaloid used for nicotine addiction treatment. It also contains pireridine, nicotine, nornicotine, citric, succinic, malic and oxalic acid. The family is considered an active ectoparasiticidal and anthelmintic [42,44,45]. The dried leaves of Nicotiana tabacum "snuff" belonging to the family Solanaceae, is considered toxic (Ed. FA VIII) and only is permitted for external use. It is used as ectoparasiticide in the form of nicotine salicylate (eudermol) or sulfate. It has also demonstrated to be useful for helminthiasis $[25,46]$, however its toxicity limits this last indication. The external use of this plant includes treatment for scabies [47] and leeches [12, 48].

The leaves, twigs, rhizomes of Podophyllum peltatum L "podophyllum" belonging to the family of Berberidáceaes is allowed only for external use [7], however in Argentina it is also use an anthelmintic (anti-worm) [49] since resin contains lignans [podophyllotoxin picropodophyllin, peltatin alpha-, beta-peltatin] heterosides glucosidices. It is well recognized in pediatric medicine as drug useful in digestive disorders. Its conservation is difficult because it's essential oil content decreases by $1 \%$ per month.

The root bark and occasionally the trunk and the fruits of Punica granatum L known as "Granado" belonging to the family Lythraceae has demonstrate action against tenias (accompanied 
by a purgative to induce expulsion of tapeworm) [11]. The decoction of the root bark is the best way to obtain useful formulations [7]. Its use was recommended for 15 centuries as an anthelmintic (especially as taenicide). It contains alkaloids (pelletierine, isopeletierina, pseudopeletierine, Nmetilpeletierina) hydrolysable tannins (3,6-hexahidrofenoilglucosa, granatinas $\mathrm{A}$ and $\mathrm{B}$, punicafolina, punicacorteínas $\mathrm{A}, \mathrm{B}$ and $\mathrm{C}$, punigluconina, punicalin, punicalagin). The harmful effects due to the fraction absorbed in the intestine, have led to quit its use and its indication rest for veterinary use. [11].

The flowering tops, stems, leaves and flowers of Deruta graveolens when extract and distilled in pot stills to steam, is known as "Ruda" (belonging to the family of Rutaceae) which gives an essential oil that has a light attributed to ketone nonylmethyl anthelmintic effect. Its odor inhibits the fifth instar Rhodnius prolixus of triatomines. That is why it is classified with a medium level validity as an anthelmintic $[12,50]$ and because of that is suggested to associate this herb with the paico and wormwood for intestinal worms [11].

Leaf infusion of young branches of Santolina chamaecyparissus popularly known as "stranded Abrotano" belonging to the family of Asteraceaes; is used to repel aphids [7]. However, it is also used as an anti-helmintic in order to eliminate intestinal parasites in children.

The flowering tops Senecio jacobaea L. popularly known as "ragwort tansy " belonging to the family of alkaloids piriolidirices Asteraceaes, contains essential oil with metilheptilcetona-pinene, cineole, l-limonene and minor amounts of methyl-n-nonilcarbinol) which gives the properties of vermicides [7,51].

Tanacetum cinararifolium popularly known as "Pyrethrum" belonging to the family Asteraceae is use as insecticide [7,26] and to eliminate parasites of pets [32].

The flowering tops of Tanacetum vulgare popularly known as "tansy tansy" also belongs to the family of Asteraceaes and has anti-helmintic properties (oil), worming and threadworm (branches, flowers and seeds) $[7,11,40]$. Tanacetum vulgare is prohibited for free sale to the public, because of its toxicity. Its use is restricted to the manufacture of pharmaceutical specialties, master formulas, officinal preparations, homeopathic stocks and research studies.

Young branches of Thuja occidentalis L popularly known as "Yours" belonging to the family of Cupressaceaes has anthelmintic [7] and insecticide activity [35, 52]. This property can refer to the contents of Alpha-thujone.

The oil obtained from the leaves and flowering tops of Thymus vulgaris L. popularly known as "Thyme" belonging to the family of Lamiaceae, contains thymol, phenol, cimol, 1-alpha pinene, beta pinene, camphene, terpinene, geraniol and caryophyllene; and it is indicated for intestinal worms. However, it use is limited because its hepatic toxicity, albuminuria, hematuria, allergic reactions and seizures (due to high doses of thymol) [7, 53].

Leaves and stems of Verbena officinalis L. popularly known as "Verbena" belonging to the family Verbenaceae, has poor information about worming activity except for Ascaris [54]. European Commission believes that the plant cannot be recommended in therapeutics use since none of its indications have been justified with clinical studies or trials are also its side effects and toxicity are still unknown [11].

\section{Conclusions}

There are many plants found along Argentine territory that are not yet been well studied. That's why this work becomes interest in order to validate them as a tool for wormers control based on scientific publications.

Herbs have been used for centuries against endoparasiticides. The regulatory agencies (FDA, ANMAT) regulated some of the plants presented in our research like Artemisia absinthium, black walnut nut, and wormwood as useful herbs with vermicide action.

Since synthetic drugs have usually undesirably impact on the biology of our ecosystems and have also a residual effect on humans that ends in overall resistance to pesticides; plants species should be considered as alternatives to conventional chemical drugs anti-parasitic treatment. 


\section{References}

[1] A.D. Penco Martín, F. Gordon Vázquez, Remedios de origen vegetal utilizados en Medicina Popular Veterinaria en la Comarca de Zafia, Revista de Estudios Extremeños. 59 (2003) 265279.

[2] Z. Farooq et al., Emnoveterinary practices for the treatment of parasitic diseases in livestock in Cholistan desert (Pakistán), Journal of Ethnopharmacology. 118 (2008) 213 -219.

[3] P. Hördegen et al., In vitro screening of six antheímintic plant products against larval Haemonchus contortus with a modifted menthyl-thiazolyltetrazolium reduction assay, Journal of Ethnopharmacology. 108 (2006) 85-89.

[4] WHO, Estrategia de la OMS sobre medicina tradicional: 2002-2005. Ginebra, Organización Mundial de la Salud, (documento WHO/EBM/TRM/20021), 2002.

[5] WHO. Directrices de la OMS sobre Buenas Prácticas Agrícolas y de Recolección (BPAR) de plantas medicinales, 2003. Information on: http://www.fitoica.com/BibliotecaALibros /OMS/OMS08.pdf

[6] E. Mandrile, Plantas medicinales que se dispensan en la Argentina (tomo I) Ed Farma, Colegio de Farmacéuticos de la Provincia de Buenos Aires, 2003.

[7] B. Vanaclocha, S. Cañigueral, Fitoterapia, Vademecum de prescrición, Editorial 4th Ed. Elsevier, Madrid, 2003.

[8] E. Mandrile y G. Bongiorno de Pfirter, Farmoplantas: Allium sativum L, Bifase. 12(3) (1999) $1-24$.

[9] J.P. Anthony, L. Fyfe, H. Smith, Plant active components - a resource for antiparasitic agents? TRENDS in Parasitology. 21(10) (2005) 44-51.

[10] G.J. Martínez, G.E. Barboza, Natural pharmacopoeia used in traditional Toba medicine for the treatment of parasitosis and skin disorders (Central Chaco, Argentina), Journal of Ethnopharmacology. 132(1) (2010) 86-100.

[11] J.A. Burgstaller, 700 Hierbas medicinales, sus propiedades terapéuticas; usos y dosis, Ed. Plus Ultra S.A., Buenos Aires, 2005.

[12] C. Lans et al., Ethnoveterinary medicines used to treat endoparasites and stomach problems in pigs and pets in British, Veterinary Parasitology. 148(3-4) (2007) 325-340.

[13] J.B. Githiori, S. Athanasiadou, S.M. Thamsborg, Use of plants in novel approaches for control of gastrointestinal helminths in livestock with emphasis on small ruminants, Vet. Parasitol. 139 (2006) 308-320.

[14] H. Kolodziej, A.F. Kiderlen, Antileishmanial activity and immune modulatory effects of tannins and related compounds on Leishmania parasitized RAW 264.7 cells, Phytochemistry. 66 (2005) 2056-2071.

[15] P. Spencer et al., Isolation and characterisation of procya- nidins from Rumex obtusifolius, Phytochem. 18 (2007) 193-203.

[16] S. Jarića et al., An ethnobotanical study on the usage of wild medicinal herbs from Kopaonik Mountain (Central Serbia), Journal of Ethnopharmacology. 111(1) (2007) 160-175.

[17] P.J. Waller et al., Plants as de-worming agents of livestock in the Nordic countries: historical perspective, popular beliefs and prospects for the future, Acta Vet. Scand. 42 (2001) 31-44.

[18] G. Álvarez Álvarez, Temas de guardia, Editorial Ciencias Médicas, La Habana, 2002. 
[19] V.M. Solís Díaz, A.L. Abarca Salinas, M. Rojas Alba, Evaluación mediante antibiogramas del efecto bactericida de Hintonia latiflora, Artemisia ludoviciana ssp. mexicana, Castela erecta ssp. texana en una cepa de Salmonella typhimurium, Universidad Autónoma del Estado de Morelos TlahuiEdu A.C. Cuernavaca, Morelos, México, 2009.

[20] J.K. Aronson, Side Effects of Herbal Medicines, Elsevier. FB Pharmacol, FFPM (Hon) Oxford, United Kingdom, 2009.

[21] L. Manfred, 7000 recetas botánicas, 1300 a base de plantas medicinales, 14ta Ed., Editorial Kier, Argentina, 1959.

[22] M. Levebre, Aromaterapia libro practico, Lasser Press Mexicana, S. A. Colonia Juárez, México D. F., 1995.

[23] J. Bruneton, Farmacognosia, Fitoquímica Plantas Medicinales, 2da Ed., Editorial Acribia, S A, Zaragoza, 2001.

[24] M. Martinez, Las plantas Medicinales de Mexico, 7ma reimpresión, Editorial Botas, México, 1997.

[25] A. Marzocca, Vademécum de Malezas Medicinales de la Argentina Indigena y exóticas, Ed. Orientación Gráfica, Buenos Aires, 1997.

[26] F.A. Roig, Flora medicinal mendocina. Las Platas medicinales y aromaticas de la provincia de Mendoza (Argentina) Universidad Nacional del Cuyo, Editorial UDIUNC, Mendoza, 2002.

[27] J.R. Vallejo Villalobos et al., Uso medicinal de Atractylis gummifera en Guadiana del Caudillo (Badajoz, España), toxicidad y especies afines. Revista de Fitoterapia. Information on: www.fitoterapia.net. 8(2) (2008) 23-29.

[28] J. de Dios Muñoz, Las Plantas medicinales de la flora de la provincia de Entre Rios, UNTUADER, Argentina, 2010.

[29] D. Lopez De Guimaraes, R.S. Neyra Llanos, J.H. Romero Acevedo, Ascariasis: Comparison of the therapeutic efficacy between Paico and albendazol in children of Huaraz, Rev. Gastroenterol. Peru. 21 (2001) 212-219.

[30] R.A. Giove Nakazawa, Traditional medicine in the treatment of enteroparasitosis, Rev. Gasteroenterol. Peru. 16 (1996) 197-202.

[31] A.U. Krettli et al., The Search for New Antimalarial Drugs from Plants Used to Treat Fever and Malaria or Plants Ramdomly Selected, Mem. Inst. Oswaldo Cruz. 96(8) (2001) 10331042 .

[32] C. Kuklinski, Farmacognosia, estudio de las drogas y suatancias medicamentosas de origen natural, Editorial Omega, Barcelona, 2003.

[33] D.W. Taura, A.H. Arzai, T. Oyeyi, Evaluation of antimobial activities of Combretum micranthum L. Journal of Pure and Applied Sciences, Department of Biological Sciences (Microbiology unit), Bayero University. 2(1) (2009) 183-185.

[34] C. Marín et al., In Vitro and in Vivo Trypanocidal Activity of Flavonoids from Delphinium staphisagria against Chagas Disease, Journal of natural products, Granada, 2011.

[35] K.M. Höld et al., Alpha-thujone (the active component of absinthe): gamma-aminobutyric acid type A receptor modulation and metabolic detoxification, Proc Natl Acad Sci USA. 97(8) (2000) 3826-3831.

[36] I.K. Park, S.C. Shin, Fumigant activity of plant essential oils and components from garlic (Allium sativum) and clove bud (Eugenia caryophyllata) oils against the Japanese termite (Reti- culitermes speratus Kolbe), J. Agric. Food Chem. 53 (2005) 4388-4392. 
[37] L.M. Pessoa et al., Anthelmintic activity of essential oil of Ocimum gratissimum Linn. and eugenol against Haemonchus contortus, Vet. Parasitol. 109 (2002) 59-63.

[38] M.K. Asha et al., Anthelmintic activity of essential oil of Ocimum sanctum and eugenol, Fitoterapia. 72 (2001) 669-670.

[39] C.O. Adewunmi et al., Ethno-veterinary medicine: screening of Nigerian medicinal plants for trypanocidal properties Drug Research and Production Unit, Faculty of Pharmacy", London, UK, 2001.

[40] P. Shima Luize et al., Effects of medicinal plant extracts on growth of Leishmania (L.) amazonensis and Trypanosoma cruzi, Rev. Bras. Cienc. Farm. São Paulo. 41(1) (2005) 42-49.

[41] E. Mandrile, G. Bongiorno de Pfirter, Farmoplantas: Eugenia uniflora, Bifase. 11(2) (1998) $20-40$.

[42] B.S. Stella, A. Bandoni, Plantas de la medicina Popular Argentina, Ed Albatros, SRL bvuenos Aires, 1978.

[43] E. Mandrile, G. Bongiorno de Pfirter, Farmoplantas: Inula helenium L., Bifase. 10(2) (1997) 41-47.

[44] J. Alonzo, C. Desmarchelier, Plantas Medicinales Autóctonas de la Argentina. Bases Científicas para su aplicación en Atención Primaria de la Salud, Editorial Fitociencias, Buenos Aires, 2006.

[45] E. Mandrile, G. Bongiorno de Pfirter, Farmoplantas: Palan-Palan (Nicotiana glauca), Bifase. 9 (1996) 5-10.

[46] M. Toursarkissian, Plantas Medicinales de la Argentina.Sus nombres botánicos, bulgares, usos y distribución geográfica, Editorial Hemisferio Sur, Argentina, 1980.

[47] J. Fajardo Rodríguez, y participantes de la Universidad Popular de Albacete, 100 flores de los campos de Albacete, Albacete, 2003.

[48] E. Blanco, M.J. Macía, R. Morales, Medicinal and veterinary plants of El Caurel (Galicia, northwest Spain), Journal of Ethnopharmacology. 65 (1999) 113-124.

[49] L.E. Ray, Podophyllum Peltatum and Observations on the Creek and Cherokee Indians: William Bartram's Preservation of Native American Pharmacology, Yale J. Biol. Med. 82(1) (2009) 25-36.

[50] F. Calzada, L. Yépez-Mulia, A. Aguilar, In vitro susceptibility of Entamoeba histolytica and Giardia lamblia to plants used in Mexican traditional medicine for the treatment of gastrointestinal disorders, Journal of Ethnopharmacology.108(3) (2006) 367-370.

[51] M.A. Bonet, J. Vallès, Ethnobotany of Montseny biosphere reserve (Catalonia, Iberian Peninsula): Plants used in veterinay medicine, Journal of Ethnopharmacology. 110 (2006) 130-147.

[52] B. Naser et al., Thuja occidentalis (Arbor vitae): A Review of its Pharmaceutical, Pharmacological and Clinical Properties, Evid Based Complement Alternat Med. 2(1) 2005) 69-78.

[53] J. Mikus et al., In vitro effect of essential oils and isolated mono- and sesquiterpenes on Leishmania major and Trypanosoma brucei, Planta Medica. 66 (200) 366-368.

[54] U. Cakilcioglu, I. Turkoglu, An ethnobotanical survey of medicinal plants in Sivrice (Elazı̆̆Turkey), Journal of Ethnopharmacology. 132(1) (2010) 165-175. 